\title{
Les tons dans les dictionnaires de langues gabonaises: situation et perspectives
}

\author{
Thierry Afane Otsaga, Département d'afrikaans et de néerlandais, Université \\ de Stellenbosch, Stellenbosch, République d'Afrique du Sud \\ (thiafane@yahoo.fr)
}

Résumé: La plupart des dictionnaires des langues gabonaises, parus jusqu'à ce jour, n'ont pas vraiment tenu compte de la tonalité dans la rédaction des articles. Or, à présent, il est unanimement reconnu que le ton joue un rôle important dans le fonctionnement des langues bantoues en général et des langues gabonaises en particulier. Les dictionnaires, outils d'apprentissage, de standardisation, de préservation et de l'emploi correct d'une langue, ne pourront plus négliger cet aspect dans leur traitement lexicographique. Malgré le grand nombre de tons qui existent dans les langues gabonaises et la difficulté de les noter tous dans la présentation des articles, il est néanmoins nécessaire de trouver des moyens de mettre en valeur chaque ton, sans néanmoins rendre trop compliqué l'emploi du dictionnaire. L'objectif de notre exposé est de formuler, pour ces dictionnaires, certaines directives qui permettront de prendre en considération la tonalité des langues gabonaises, tout en gardant accessible l'emploi du dictionnaire. Avant de formuler ces directives, notre exposé tente tout d'abord de clarifier le problème par l'analyse et l'énumération des différents tons identifiés jusqu'à ce jour dans les langues gabonaises. En outre, nous prenons en considération certains points de vue qui, dans les dictionnaires, ont voulu identifier les tons, en donnant des exemples du traitement de la tonalité dans certains dictionnaires des langues tonales autres que les langues gabonaises.

Mots-clés: DICTIONNAIRES, LANGUES GABONAISES, TONS, TONALITÉ, RÉDACTION DES ARTICLES, PERSPECTIVES, LANGUES BANTOUES, TRAITEMENT LEXICOGRAPHIQUE, OUVRAGES DE RÉFÉRENCE LEXICOGRAPHIQUE, COURBE MÉLODIQUE, SIGNES DIACRITIQUES, ORTHOGRAPHE, PRONONCIATION

\footnotetext{
Abstract: Tones in Dictionaries of the Gabonese Languages: State and Perspectives. Until now most dictionaries that have appeared in the Gabonese languages have not really taken tonality into account in the editing of articles. But at present it is unanimously recognized that tone plays an important role in the functioning of Bantu languages in general and Gabonese languages in particular. Dictionaries which are tools in the learning, standardization, preservation and correct usage of a language can no longer neglect this aspect in their lexicographic treatment. In spite of the large number of tones present in the Gabonese languages and the difficulty of attending to all of them in the presentation of the articles, it is nevertheless necessary to find means to account for each tone, without however rendering the use of the dictionary too complicated. The objective of this article is to formulate certain directions for these dictionaries, which
} 
will make allowance for the tonality of the Gabonese languages, keeping the dictionary completely accessible to users. After having formulated these directions, our article firstly attempts to clarify the problem by analysing and enumerating the different tones identified in the Gabonese languages up till now. Moreover, we consider certain angles from which, in dictionaries, tones can be identified, by giving examples of the treatment of tonality in certain dictionaries of tonal languages other than the Gabonese languages.

Keywords: DICTIONARIES, GABONESE LANGUAGES, TONES, TONALITY, EDITING OF ARTICLES, PERSPECTIVES, BANTU LANGUAGES, LEXICOGRAPHIC TREATMENT, LEXICOGRAPHIC REFERENCE WORKS, MELODIC CURVE, DIACRITIC SIGNS, ORTHOGRAPHY, PRONUNCIATION

\section{Introduction}

Les langues gabonaises sont dans leur ensemble des langues bantoues et comme la plupart des langues bantoues elles comportent des éléments suprasegmentaux telles que l'accent, l'intonation, la longueur, les tons, etc. qui jouent un rôle important dans le fonctionnement de ces langues. De tous ces éléments suprasegmentaux, les tons sont, sans conteste, ceux dont l'implication est le plus déterminant dans le comportement des dites langues (Afane Otsaga 2001: 155). Les tons sont des oppositions de hauteurs qui dans certaines langues sont utilisées comme unités distinctives (Baylon et Fabre 1990: 101). Tous les travaux de description réalisés sur les langues gabonaises reconnaissent unanimement le rôle majeur que jouent les tons dans celles-ci. De ces différents travaux, il ressort que les langues gabonaises sont non seulement des langues à tons puisqu'elles utilisent la hauteur mélodique à des fins distinctives, mais sont aussi des langues à type tonal parce que la structure tonale de certains termes varient selon qu'ils sont en isolation ou en contexte. Pour ce qui concerne les travaux lexicographiques sur les langues gabonaises, particulièrement pour la réalisation des dictionnaires, une question importante demeure sans réponse: doit-on oui ou non noter les tons dans les dictionnaires de langues gabonaises? L'objectif de cet article est non seulement de faire le point en ce qui concerne le traitement de la tonalité dans les dictionnaires de langues gabonaises jusqu'à présent, mais surtout de proposer quelques méthodes qui pourraient aider à résoudre ce problème dans le traitement lexicographique des langues gabonaises.

\section{Un mot sur les tons dans les langues gabonaises}

Les tons sont généralement répartis en deux catégories. D'une part les tons ponctuels où seul un aspect de la courbe mélodique est tenu en compte (le plus haut ou le plus bas) ainsi que les tons modulés qui se distinguent par des directions successives de la courbe mélodique (Baylon et Fabre 1990: 101). Dans 
les langues à tons, la variation mélodique est généralement associée à une syllabe (Matthews 1997: 379).

Les différentes descriptions ${ }^{1}$ réalisées jusqu'à ce jour sur les langues gabonaises ont identifié dans celles-ci l'existence de trois catégories de tons.

\section{Les tons principaux}

Par tons principaux, j'entends les tons qui sont les plus récurrents et les mieux répartis dans les langues gabonaises. Il s'agit

- du ton ponctuel haut noté avec un accent aigu ('). C'est le point le plus haut de la courbe mélodique.

$\begin{array}{lll}\text { Langues } & \text { Exemples }^{2} & \text { Traduction française } \\ \text { Fan } & \text { mvám } & \text { générosité } \\ \text { Meka } & \text { nké } & \text { plantation } \\ \text { Yipunu } & \text { mvúlà } & \text { pluie }\end{array}$

— du ton ponctuel bas noté avec un accent grave ( ` ). C'est le point le plus bas de la courbe mélodique.

$\begin{array}{lll}\text { Langues } & \text { Exemples } & \text { Traduction française } \\ \text { Gisira } & \text { bìlìmè } & \text { les années } \\ \text { Ikota } & \text { èlùngá } & \text { panier } \\ \text { Latege } & \text { jùrù } & \text { tirer }\end{array}$

— du ton modulé montant noté avec un accent circonflexe inversé ( ${ }^{u}$ ). Il s'agit d'une courbe mélodique qui prend deux directions successives. Elle descend d'abord vers le point le plus bas, avant de remonter vers le point le plus haut.

$\begin{array}{lll}\text { Langues } & \text { Exemples } & \text { Traduction française } \\ \text { Séki } & \text { dikŏn } & \text { lance } \\ \text { Sake } & \text { yă } & \text { marécage } \\ \text { Yilumbu } & \text { dĭmbú } & \text { village }\end{array}$

— du ton modulé descendant noté avec un accent circonflexe ( ${ }^{\wedge}$ ). Il s'agit d'une courbe mélodique qui prend deux directions successives. Elle monte d'abord vers le point le plus haut, avant de redescendre vers le point le plus bas.

$\begin{array}{ll}\text { Langues } & \text { Exemples } \\ \text { Lekanini } & \text { yầ } \\ \text { Liwanzi } & \text { tô̂lộ } \\ \text { Omyenen } & \text { íbôbò }\end{array}$

Traduction française autrefois sommeil peur 


\section{Les tons intermédiaires}

Par tons intermédiaires, j'entends les tons qui sont moins récurrents et moins répartis dans les langues gabonaises que les tons principaux, mais dont la présence dans ces langues n'est pas négligeable puisque facilement remarquable. Il s'agit

- du ton ponctuel moyen noté avec une barre horizontale $\left({ }^{-}\right)$. C'est le point situé au milieu de la courbe mélodique, c'est-à-dire entre le point le plus bas et le point le plus haut de la courbe mélodique.

\begin{tabular}{|c|c|c|}
\hline Langues & Exemples & Traduction française \\
\hline & āsōon & dent \\
\hline Liwanzi & jìwānī & frapper \\
\hline Omyene & tsólōo & oiseau \\
\hline
\end{tabular}

— du ton haut-abaissé noté avec une barre verticale ( ' ). Notons que ce ton ne se place pas au-dessous d'un segment syllabique comme c'est généralement le cas des tons et est aussi appelé faille tonale. La faille tonale a pour résultat de produire des tons que l'on appelle haut-abaissé. Ces tons sont réalisés phonétiquement à un niveau qui peut être assimilable au ton moyen, mais contrairement à un véritable ton moyen, ils ne peuvent être suivis par un ton plus haut (Hombert 1990: 102). Ce ton est suivi d'un terrassement tonal, c'est-à-dire que la série de tons hauts qui suit la faille tonale est réalisée au même niveau que le ton haut-abaissé.

$\begin{array}{lll}\text { Langues } & \text { Exemples } & \text { Traduction française } \\ \text { Fan } & \text { áwi'ny } & \text { tuer } \\ \text { Lembaama } & \text { mèma'áta' } & \text { j'ai tiré } \\ \text { Ngubi } & \text { íngu'yí } & \text { mère }\end{array}$

- du ton supra-haut noté avec deux accents aigus ("). Ce ton a pour point de départ une valeur plus élevée qu'un ton haut et qui ensuite chute très rapidement (Hombert 1990: 103).

$\begin{array}{lll}\text { Langues } & \text { Exemples } & \text { Traduction française } \\ \text { Yipunu } & \text { éläbì } & \text { il voit } \\ \text { Lembaama } & \text { vúghi } & \text { miel } \\ \text { Lekaniñi } & \text { sâsì } & \text { appât }\end{array}$

\section{Les tons secondaires}

Par tons secondaires, j'entends les tons assez rares dans les langues gabonaises et dont la répartition entre ces dernières est véritablement infime. Il s'agit 
- du ton infra-bas noté par deux accents graves ( " ). Sa définition est identique à celle du ton supra-haut, avec la différence que sa courbe est descendante. Il s'agit donc d'un ton bas réalisé plus bas que le ton bas normal. Il existe parfois un contraste entre le ton bas ponctuel et le ton infra-bas. Il est fréquent que cette distinction ne se produise que dans certains contextes, par exemple avant la pause (Hombert 1990: 102).

$\begin{array}{lll}\text { Langues } & \text { Exemples } & \text { Traduction française } \\ \text { Lenduma } & \text { wülú } & \text { pied } \\ \text { Meka } & \text { wümá } & \text { combattre }\end{array}$

- du ton très haut descendant noté par un accent aigu suivi d'un accent circonflexe ('^). Je n'ai pas de définition précise de ce ton, mais on pourrait penser qu'il s'agit d'un ton supra-haut qui chute par la suite. Le seul exemple que j'ai de ce ton vient du Yipunu.
Langue
Exemple
Yipunu
ngáânzì
Traduction française racines

Le recensement des tons révélés ainsi dans les langues gabonaises n'est certainement pas exhaustif, car toutes les langues gabonaises n'ont pas encore été décrites. Mais il permet de constater qu'il en existe un grand nombre et que par conséquent leur influence dans le fonctionnement de ces langues est sans conteste à prendre en compte.

\section{Le traitement de la tonalité dans les dictionnaires existants}

Les premiers travaux lexicographiques dans les langues gabonaises ont été exécutés par des anciens administrateurs coloniaux et par les missionnaires. L'objectif premier de ces œuvres à caractère lexicographique (glossaires, lexiques, dictionnaires ...) était d'établir une communication efficace entre ces administrateurs et missionnaires, et les populations gabonaises (Mavoungou 2001). De même, les réalisateurs de ces travaux n'étaient ni des linguistes de formation, encore moins des lexicographes attitrés. Malgré ce fait, ces travaux, dans leur globalité, répondent effectivement aux normes métalexicographiques requises (Mihindou 2001: 161), bien que certaines améliorations y soient nécessaires.

Bien que n'ayant pas eu à ma disposition tous les dictionnaires ${ }^{3}$ réalisés sur les langues gabonaises, je peux estimer que ceux que j'ai pu consulter constituent entre 70 et $80 \%$ environ de ce qui existe. De cette consultation, j'ai pu remarquer, en ce qui concerne le traitement de la tonalité, les situations suivantes:

- Deux tiers des dictionnaires consultés ne font pas état de la tonalité. Les articles des dictionnaires sont présentés sans structure tonale et cette 
absence n'est, ni mentionnée, ni expliquée dans les introductions (prétextes) des dictionnaires. La majorité de ces dictionnaires sont plutôt des glossaires ou des lexiques, car il s'agit dans l'ensemble de listes alphabétiques de mots dans une langue-source suivie de leurs traductions équivalentes dans une langue-cible. Le Dictionnaire Français-Yipounou / Yipounou-Français (1966) de l'Église Évangélique du Gabon est une très bonne illustration de cette situation. Dans ce dernier aucun ton n'est noté, alors que le Yipunu est une langue à tons avérée.

- Le deuxième scénario est celui des dictionnaires qui utilisent des diacritiques dans la transcription. Mais ces diacritiques sont utilisés non pas comme marques de tonalités, mais plutôt comme éléments des symboles alphabétiques utilisés. Le Dictionnaire Français-Mpongwé, suivi d'éléments de grammaire (1995) de Raponda-Walker nous donne une excellente illustration de ce type de démarches. Dans ce dernier par exemple le «è » transcrit le son phonétique [ $[\varepsilon]$, alors que le «ó » y figure pour transcrire le son phonétique [॰]. En d'autres termes, les tons n'y sont pas pris en considération. Mais contrairement au premier cas, celui-ci a la particularité de prendre déjà en compte certaines situations d'intonation ou d'accentuation en ce qui concerne la prononciation du «è » et du «ó ».

— Le dernier scénario est à l'image du second, car il ne tient compte que des phénomènes d'intonation et d'accentuation qui sont clairement mis en évidence au niveau de la transcription orthographique. Or, nous pouvons constater que dans ces dictionnaires, plusieurs traits accentuels (ou d'intonation) attribués aux langues gabonaises sont en réalité des manifestations tonales. Ce qui peut s'observer dans l'Encyclopédie Pahouine (1901) de V. Largeau où ce dernier présente par exemple trois réalisations différentes $\mathrm{du}$ « $\mathrm{a}$ » dans la Fan $(\mathrm{a}, \overline{\mathrm{a}}, \hat{\mathrm{a}})$. Le « $\mathrm{a}$ » sans accent pour lui est équivalent à celui qui existe en français comme dans «ami». Celui avec la barre horizontale « $\overline{\mathbf{a}}$ » est décrit comme un son ayant la même qualité que le premier, mais avec une quantité plus importante, alors que celui avec l'accent circonflexe " $\hat{a}$ " augmente aussi bien sur le plan qualitatif que quantitatif et se prononce comme le «â" français dans "pâte ». Cette même application est faite pour d'autres sons comme «e » ou « $\mathrm{O} »$. Il semble que Largeau, qui était un administrateur colonial et non pas un linguiste ou lexicographe de formation, confond l'intonation, l'accentuation et la tonalité en Fan. Les descriptions intonatives ou accentuelles qu'il donne de ces sons correspondent en réalité aux situations où ils portent le ton bas, moyen, haut, montant ou descendant comme mis en évidence dans différentes descriptions du Fan par Andeme Allogo (1980), Voltz (1990), Cinnamon (1990), Ondo Mebiame (1992), etc. 
La confusion de Largeau entre tons et d'autres phénomènes suprasegmentaux a pu se glisser dans d'autres dictionnaires qui, j'insiste sur ce point, ont été réalisés (pour la plupart) par des non-spécialistes en matière de description linguistique. Cela peut donc expliquer l'absence des tons dans le plus grand nombre de dictionnaires réalisés dans les langues gabonaises.

Signalons également que dans un petit nombre de dictionnaires et d'ouvrages $^{4}$ (tel que le dictionnaire Fang-Français en Français-Fang de Galley 1964) réalisés dans les langues gabonaises, les tons sont notés non pas sur les segments, mais entre parenthèse à côté de ces derniers. Cette méthode donne l'impression que pour les réalisateurs de ces ouvrages, les tons ne font pas parties intégrantes de la langue et sont des phénomènes situationnels qui apparaissent ça et là dans le fonctionnement de celles-ci.

\section{Quelques avis informels au sujet de la notation des tons dans les dic- tionnaires}

Le point de vue le plus général rencontré chez beaucoup d'auteurs en ce qui concerne l'absence de tons dans les dictionnaires de langues gabonaises est que ceux-ci ne sont pas indispensables. La raison souvent avancée est que les premiers ouvrages à caractère lexicographique ne tenaient pas compte des tons de façon générale, mais ils ont étés les principaux supports qui ont aidé beaucoup de missionnaires et d'administrateurs coloniaux à apprendre les langues gabonaises. Dans mes discussions sur ce problème avec certains locuteurs gabonais profanes de la linguistique, la plupart m'ont ressorti l'exemple des prêtres européens qui disaient les messes dans les langues gabonaises, sans pourtant avoir une prononciation tonale très correcte de ces langues. Pour eux une langue peut d'abord s'acquérir sur le plan segmental, la structure suprasegmentale de celle-ci s'acquérant par la pratique régulière au contact des locuteurs.

Je voudrais rappeler à ceux qui parlent de l'expérience des prêtres européens, qu'ils ne doivent pas non plus oublier les incidents survenus dans certaines églises du Gabon au cours de messes dites par ces derniers. L'incident le plus courant était que les fidèles sortaient de l'église parce qu'ils se sentaient choqués ou insultés par les propos d'un prêtre qui en prononçant mal certains termes (sans la bonne tonalité) aurait signifié des choses qu'il ne souhaitait pas dire en réalité. Cela montre que la tonalité est primordiale dans les langues gabonaises et que par conséquent ne pas en tenir compte dans les dictionnaires pourrait occasionner, pour les apprenants de ces langues, des incidents identiques à ceux dont je viens de faire état.

Pour autant, je ne suis pas totalement en désaccord avec ceux qui pensent que l'acquisition d'une langue se réalise d'abord au niveau de sa structure segmentale, car elle est la plus palpable, autant sur le plan acoustique que sur le plan visuel. Le dictionnaire doit d'abord mettre l'accent sur la transcription (orthographique) simple et accessible des formes segmentales de la langue. Par conséquent, je ne partage pas l'avis de ceux qui estiment qu'un livre d'appren- 
tissage linguistique qui ne fait pas état des marques de tonalité est à rejeter complètement ${ }^{5}$. J'estime que la présentation de la structure segmentale est une étape ou une partie de l'entièreté structurale (segmentale et suprasegmentale) d'une langue. Il n'y a donc pas de raison à ce qu'elle soit rejetée. Beaucoup de descriptions purement linguistiques sur les langues gabonaises se restreignent bien souvent aux aspects phonétiques, phonologiques et morphologiques. Rares sont celles qui font des descriptions sémantiques ou syntaxiques de ces langues. Mais tout le monde est d'accord pour reconnaître que ces descriptions, qui ne mettent en évidence que certains aspects de la langue, sont des travaux scientifiquement valables. Présenter une langue sans sa structure tonale devrait être vu dans le même esprit. C'est un aspect de la langue qui est présenté (la structure segmentale), il faut donc l'accepter comme tel.

Pour autant, dans le cas des langues à types tonals comme le sont certaines langues gabonaises, il ne serait pas inutile pour l'usager du dictionnaire d'ajouter les marques tonales afin de faciliter la tâche aux apprenants de ces langues. $\mathrm{Au}$ lieu que l'apprenant d'une langue attende d'être au contact des usagers de celle-ci pour la pratiquer et ainsi acquérir sa structure suprasegmentale, il pourrait l'apprendre directement à travers le dictionnaire ou du moins l'avoir à sa disposition dans ce dernier. Mon avis est que les tons devraient être présentés dans les dictionnaires de langues gabonaises, mais cette présentation devrait se faire de façon méthodique et en tenant compte d'un certain nombre de paramètres.

\section{Les tons et l'orthographe}

Le problème le plus important des langues gabonaises en ce moment est leur transcription orthographique. Tous les travaux réalisés sur ces langues sont transcrits, soit phonétiquement, soit avec des symboles orthographiques différents les uns des autres. Aucune langue au monde ne s'est développée sans une forme orthographique unique utilisée par tous (Afane Otsaga 2001: 156). L'orthographe rappelons-le est l'art et la technique de la formation des mots par les lettres selon l'usage accepté (Gove 1961). Le choix d'utiliser une forme orthographique spécifique pour l'écriture d'une langue donnée est une convention qui naît d'un accord (généralement entre experts) et qui est accepté (ou imposé dans certains cas) pour être utilisé dans les écoles, les administrations (gouvernementales et non gouvernementales), la presse et tous les autres champs d'utilisation de la langue (Mdee 1999: 123). L'acceptation d'une forme scripturale d'une langue se fait généralement par la création d'un modèle d'imitation et la promotion de ce modèle.

Dans la situation des langues gabonaises, l'acceptation du modèle orthographique proposé au cours d'un colloque d'experts en avril 1999 à Libreville sous l'égide du Ministère de l'Éducation Nationale (Afane Otsaga 2001: 156) permettra de faire un pas important vers la lecture et l'écriture de ces langues par le plus grand nombre. En effet, tout débat sur la mise en valeur ou non des 
tons dans la transcription des langues gabonaises resterait vain tant que cellesci n'auraient pas une forme orthographique acceptée et utilisée par tous. Toute notation de tons serait inutile si elle ne servait pas à aider l'usager dans l'amélioration de la prononciation. Mais si ce dernier ne se retrouve même pas dans la structure segmentale (par la forme orthographique) des termes qui lui sont proposées, il lui serait difficile d'avoir accès à la structure suprasegmentale de ceux-ci. La structure segmentale d'une langue est le support de sa structure suprasegmentale. Si le support est instable, ce qu'il supporte l'est aussi forcément. Le problème de tonalité dans l'écriture des langues gabonaises ne peut donc trouver de solution tant que celui de l'orthographe n'est pas résolu.

Je pourrais ajouter que la vulgarisation de l'orthographe de langues gabonaises devrait aussi inclure la vulgarisation des marques de tonalité. Un certain nombre de diacritiques (pas trop nombreux) pourrait être retenu pour transcrire les tons les plus réguliers et les plus répandus dans les langues gabonaises. La promotion de ces diacritiques devrait se faire en même temps que les symboles retenus pour l'orthographe. Cette promotion consistera essentiellement à habituer les usagers à l'utilisation de ces diacritiques, mais surtout à leur permettre d'associer clairement chaque diacritique à un ton spécifique, en précisant la manière dont ce ton doit être réalisé.

\section{Quelques exemples de notation des tons dans les dictionnaires de langues bantoues}

Les langues gabonaises font parties de l'ensemble bantou. Toutes les langues appartenant à ce groupe rencontrent le même problème en ce qui concerne la prise en compte de la tonalité dans les dictionnaires. Ce problème est abordé de différentes façons selon le choix des lexicographes.

La situation la plus courante est celle des dictionnaires qui présentent les marques de tons dans la macrostructure du dictionnaire, mais sans donner aucune explication ni par rapport à la présence de ces tons, ni par rapport à leur rôle dans le fonctionnement de la langue. C'est la situation générale que nous avons observée pour les dictionnaires des langues gabonaises. Deux hypothèses peuvent être émises par rapport à cette attitude:

- La première hypothèse est que certains lexicographes prétendent que puisque les usagers-cible des dictionnaires sont des locuteurs natifs de ces langues, par la maîtrise intuitive qu'ils ont de la langue, ils n'ont pas besoin d'explications particulières sur la structure suprasegmentale d'une langue qu'ils maîtrisent déjà. C'est l'occasion ici de rappeler à ceux qui pourraient se reconnaître dans cette hypothèse que le dictionnaire même s'il est réalisé avant tout selon les besoins du groupe-cible, peut dans certaines situations tomber entre les mains d'un individu n'appartenant pas au groupe-cible du dictionnaire, comme c'est souvent le cas aujourd'hui dans un monde de plus en plus polyvalent. Les informations 
sur la structure suprasegmentale pourraient, dans ce cas, être utiles à un non-locuteur de la langue.

- La deuxième hypothèse est que certains lexicographes travaillant sur les langues bantoues estiment que dans les dictionnaires des langues indo-européennes, qui ont pourtant une longue tradition lexicographique, aucune explication particulière n'est faite au sujet des phénomènes suprasegmentaux comme l'accent ou la longueur par exemple. Par rapport à cet argument, je voudrais souligner que les langues indo-européennes ont une très longue tradition écrite. Les problèmes liés à l'orthographe et à la prononciation ont depuis longtemps trouvé des solutions dans ces langues, contraire aux langues bantoues qui se cherchent encore sur tous les plans. Les phénomènes accentuels en français par exemple sont clairement identifiés par un certain nombre de diacritiques qui ôtent toute ambiguiité dans la prononciation de cette langue. La comparaison entre les dictionnaires des langues indo-européennes et les dictionnaires des langues bantoues est donc une méprise qu'il est souhaitable d'éviter.

Dans d'autres dictionnaires, les lexicographes ont choisi de présenter la structure tonale de façon complète et sans restriction telle qu'elle apparaît dans les termes de la langue. Tous les tons y sont marqués. C'est le cas du dictionnaire Tsivenda ${ }^{6}$-English (Van Warmelo 1937) dans lequel les tons hauts, bas, moyens et haut abaissés sont notés. Selon les rédacteurs de ce dictionnaire, ces quatre tons ont été retenus parce qu'ils sont les seuls qui apparaissent dans les termes en isolation, les autres tons étant de simples variations de ces derniers en situation de discours. Ils représentent donc la tonalité de base de la langue, c'est pourquoi ils sont les seuls à être notés dans le dictionnaire. Les tons de base sont ainsi notés au niveau des lemmes à tous les niveaux où ils apparaissent.

Cette présentation, bien que visant l'exhaustivité, a l'inconvénient de trop alourdir les articles du dictionnaire par la présence d'un grand nombre de signes diacritiques. La vue d'un trop grand nombre de signes, qu'ils ne maîtrisent pas forcément, est décourageant pour beaucoup d'usagers de dictionnaires.

Un autre cas est celui du dictionnaire English-Zulu ${ }^{7}$ et Zulu-English (Doke, Malcolm, Sikakana et Vilakazi 1990) dans lequel il est indiqué que fondamentalement le Zulu est une langue qui comporte neuf variétés tonales. Mais les rédacteurs estiment (comme ceux du dictionnaire Venda) que seuls les tons ponctuels haut et bas sont les véritables tons de la langue; les autres sont des variations et/ou des associations de ces deux tons. Mais à la différence du dictionnaire Venda où les marques de tonalité sont bien visibles dans la présentation de la macrostructure, le dictionnaire Zulu se contente de décrire, dans les pré-textes, les différents contextes d'apparition ou de prononciation de chaque ton. Le ton descendant par exemple est décrit comme étant le résultat d'un ton haut qui est articulé avec une dépression occasionnée généralement en Zulu par la présence des sons tels que $b h, d, g, g c, g x, g q, v, z, d l, h h$ et $j$. Dans ce dic- 
tionnaire aucune tonalité n'est présentée dans la macrostructure. L'usager doit donc régulièrement consulter les pré-textes pour comprendre le comportement de la langue au niveau suprasegmental.

Cette méthode, bien qu'allégeant la macrostructure du dictionnaire en faisant une économie en terme d'espace (dans le dictionnaire) et de temps (pour le travail du dictionnaire), a l'inconvénient de ne présenter aucun ton dans la macrostructure. Toutes les explications relatives aux tons sont concentrées dans les pré-textes. Cela risque de ne pas être très utile aux usagers, surtout que ces derniers n'ont pas toujours la culture de la bonne utilisation du dictionnaire et par conséquent ils consultent assez peu ou rarement les pré-textes.

\section{Perspectives}

Le problème de la tonalité dans les dictionnaires est donc traité différemment selon le choix des lexicographes. Pour ce qui est des dictionnaires de langues gabonaises, la problématique reste entière, puisque n'ayant pas encore été véritablement abordée par les spécialistes (linguistes et lexicographes). Le Gabon est entré dans un processus irréversible de développement de ses langues. Ce processus a comme priorités les plus immédiates, la réalisation de dictionnaires dont le contenu devrait non seulement satisfaire les besoins des usagers, mais prévoir les problèmes de ces derniers. Il ne fait aucun doute que l'une de ces difficultés serait la réalisation tonale. Il n'est donc pas inutile dès aujourd'hui de proposer des voies qui pourraient aider à mieux aborder le problème de tons dans les futurs dictionnaires de langues gabonaises.

\section{Quelques propositions}

L'un des problèmes majeurs rencontré dans les langues gabonaises est celui de la pluralité des tons. La trop grande variété de tons dans les langues gabonaises est l'une des principales raisons de la réticence d'un grand nombre de chercheurs à les noter dans les dictionnaires ou des ouvrages à référence lexicographique. Les noter non seulement alourdirait les textes, mais on n'est jamais certain de pouvoir tous les noter convenablement comme ils apparaissent réellement dans les langues. L'une des solutions que l'on pourrait proposer est que pour la notation des tons dans les dictionnaires et autres ouvrages à référence lexicographique, on se limite aux tons les moins récurrents et à deux tons au maximum. Cette option ferait appel à une description linguistique ${ }^{8}$ détaillée de la langue choisie. En vue de la description tonologique de cette langue, le lexicographe pourra clairement y distinguer la fréquence des différents tons. Ma proposition est donc que seuls les tons les moins fréquents soient notés. Mais la prise en compte des tons à noter doit se faire en fonction de chaque terme. Si un terme n'a que deux types de tons, seul un ton doit être noté. Mais si le terme contient plus de trois tons, seuls deux devraient être notés. La notation des tons 
ne devrait pas aller au-delà de deux. Cela permettra de ne pas trop surcharger les articles dans le dictionnaire, à condition que le lexicographe informe les usagers de cette disposition dans les pré-textes.

La solution de noter les tons les moins fréquents ne pourrait être efficace que si la variété des tons retenus est minime. S'il existe plus de trois variétés tonales dans une langue, il serait difficile pour les usagers de deviner quels sont les tons qui ne sont pas notés et comment les différencier de ceux qui ne le sont pas. La solution à cette situation serait que seuls les deux tons les plus fréquents de la langue soient retenus. Les descriptions linguistiques permettraient d'identifier les deux tons les plus réguliers dans chaque langue. De ces deux tons, le moins régulier devrait être noté dans le dictionnaire.

Une autre approche serait de ne tenir compte que des tons ponctuels principaux (haut, bas et moyen). Il semble que dans la majorité des langues à tons, le ton haut et le ton bas soient les plus faciles à identifier sur le plan auditif, leur courbe mélodique étant constante et courte. Ainsi, le ton moyen qui n'est pas aussi facile à identifier que les deux autres, serait obligatoirement noté accompagné par le moins fréquent des deux autres (le haut ou le bas). Dans cette configuration, seuls deux tons seraient notés, tout en ayant la certitude que les articles du dictionnaire ne seraient pas trop surchargés par les marques tonales. Cette démarche s'inspire en partie de celle adoptée dans les dictionnaires Venda et Zulu dans lesquels les autres tons (montant, descendant, failletonale, etc.) sont considérés comme de simples variations des tons ponctuels haut et bas.

Marquer les tons dans un dictionnaire peut aussi se faire d'une autre manière que par les signes diacritiques habituels. Dans le cas où le lexicographe estime ne pas vouloir (ou pouvoir) se passer de certains tons, il pourrait, comme je l'ai proposé plus tôt, identifier les deux tons les plus réguliers et ne noter que le moins fréquent des deux. À cela il pourrait ajouter la transcription en italique par exemple, des syllabes portant un autre ton qu'il estime important. Le caractère italique serait ainsi une marque tonale, mais à la différence qu'il ne s'agirait pas d'une diacritique spécifique. Une fois de plus, l'objectif est de marquer le mieux possible la structure suprasegmentale des langues gabonaises, sans pour autant rendre le dictionnaire illisible ou décourageant pour les usagers.

\section{Conclusion}

L'intérêt pour les dictionnaires en langues gabonaises va de pair avec le développement de ces langues. Les principaux problèmes auxquels sont confrontés les lexicographes travaillant sur ces langues, est celui de l'orthographe et de la notation de la tonalité. L'objectif de cet exposé était de proposer quelques voies en vue d'aider à résoudre le problème de la notation des tons dans les dictionnaires de langues gabonaises. Ces propositions ne sont pas exhaustives et ne prétendent pas être les seules possibles pour la résolution du problème, mais 
elles pourraient fournir une issue qui permettrait de mieux tenir compte, dans l'avenir, de la tonalité dans les dictionnaires de langues gabonaises, contrairement à ce qui s'est fait jusqu'à ce jour.

Il est important de souligner que, quelles que soient les démarches choisies par les lexicographes en ce qui concerne la notation des tons dans les dictionnaires, celles-ci doivent être expliquées en détails dans les pré-textes afin d'informer les usagers et leur permettre d'utiliser au mieux le dictionnaire. Il est évident que sans ces explications, toute démarche serait vouée à l'échec, du moins en ce qui concerne l'objectif de permettre aux usagers de mieux saisir la bonne prononciation tonale des langues gabonaises.

\section{Notes}

1. Les références de ces descriptions sont disponibles dans la bibliographie.

2. Les exemples sont transcrits en orthographe des langues gabonaises proposée à Libreville par un colloque d'expert en avril 1999.

3. Les titres de tous les dictionnaires de langues gabonaises consultés sont disponibles dans la bibliographie.

4. Ces dictionnaires et ouvrages parcourus il y a longtemps n'étaient pas à ma disposition au moment où j'ai rédigé cet article.

5. Une polémique existe au Gabon entre certains linguistes et la Fondation Raponda Walker auteur des livres «Rapido-langue». Les linguistes estiment que ces ouvrages ne sont pas scientifiques parce que la tonalité n'y est pas marquée, alors que la Fondation Raponda pense que mettre les tons pourrait rendre les ouvrages difficilement accessibles pour le commun des usagers non-initié à la linguistique.

6. Le Tsivenda est une langue bantoue d'Afrique du Sud et s'écrit généralement avec un accent circonflexe renversé en dessous $\mathrm{du}$ « $\mathrm{d}$ ». Cette diacritique n'a malheureusement pas pu être reproduite, mais cette absence ne chance pas grand chose pour sa prononciation.

7. Le Zulu est une langue bantoue d'Afrique du Sud.

8. Une collaboration plus étroite et plus efficace entre linguistes et lexicographes gabonais reste, dans ce contexte, plus que nécessaire. Les lexicographes pourront se servir des résultats des descriptions linguistiques pour améliorer le traitement lexicographique des langues gabonaises.

\section{Bibliographie}

Adam, J.J. 1969. Dictionnaire Ndumu-Mbebe-Français et Français-Ndumu-Mbede. Petite flore de la région de Franceville (Gabon). Grammaire Ndumu-Mbede. Archevêché de Libreville (Bar-le-Duc): Imprimerie St. Paul.

Afane Otsaga, T. 2001. Les dictionnaires bilingues dans les langues gabonaises: Approche méthodlogique. Éléments de lexicographie gabonaise, Tome I: 137-159. New York: Jimacs-Hillman.

Andeme Allogo, M.F. 1980. Esquisse Phonologique du Nzaman de Makokou. Mémoire de Licence. Libreville: Université Omar Bongo. 
Baylon, C. et P. Fabre. 1990. Initiation à la linguistique. Paris: Nathan

Bessieux, J.R. (Mgr). 1847. Dictionnaire Français-Pongoué / Pongoué-Français. 2 volumes. Amiens: Lenoël-Hereaurt.

Biton, A. 1907. Dictionnaire Français-Ndumu et Ndumu-Français, précédé d'éléments de grammaire. Nantes: Imprimerie Bourgeois.

Cinnamon, J. 1990. Fay (Nzaman). Reoue Gabonaise des Sciences de l'Homme 2: 175-188.

Doke, C.M., D.M. Malcolm, J.M.A. Sikakana et B.W. Vilakazi. 1990. English-Zulu / Zulu-English Dictionary. Johannesburg: Witwatersrand University Press.

Église Évangélique du Gabon. 1960. Dictionnaire Français-Yipounou / Yipounou-Français. Mouila.

Fishman, Joshua A. (Éd.). 1968. Readings in the Sociology of Language. La Haye/Paris: Mouton.

Galley, S. 1964. Dictionnaire fang-français et français-fang, suivi d'une grammaire fang. Neuchâtel: Henri Messeiller.

Gove, P.B. (Éd. en chef). 1961. Webster's Third New International Dictionary of the English Language. London: G. Bell/ Springfield, Mass.: G. \& C. Merriam.

Hombert, J.M. 1990. Problèmes phonétiques et phonologiques rencontrés dans les langues du Gabon. Revue Gabonaise des Sciences de l'Homme 2: 97-103.

Kwenzi-Mikala, J.T. 1980. La phonologie du Yipunu. Éléments de description du Yipunu. Lyon: CRLS. Univ. Lyon II.

Largeau, V. 1901. Encyclopédie Pahouine. Éléments de grammaire et dictionnaire français-pahouin. Paris: E. Leroux.

Lejeune, L. 1892. Dictionnaire français-fang ou pahouin, précédé de quelques principes grammaticaux sur cette même langue. Paris: A Faivre/H. Teillard.

Marichelle, C. 1900. Dictionnaires Français-Vili. Loango.

Marling (R.P.). 1872. Dictionnaire fang-français. New York.

Martrou, L. 1924. Lexique fãn-français. Paris: Procure Générale (des Pères du St Esprit) / Abbeville: Imprimerie Paillard.

Matthews, P.H. 1997. Concise Dictionary of Linguistics. New York: Oxford University Press.

Mavoungou, P.A. 2001. La mondialisation et la lexicographie trilingue ou plurilingue au Gabon. Éléments de lexicographie gabonaise, Tome I: 160-183. New York: Jimacs-Hillman.

Mdee, J.S. 1999. Dictionaries and the Standardization of Spelling in Swahili. Lexikos 9: 119-134.

Mihindou, G.R. 2001. Apports des missionnaires à la lexicographie gabonaise: Dictionnaires bilingues Fang-Français/Français-Fang; Français-Yipounou/Yipounou-Français; FrançaisMpongwé/Mpongwé-Français. Éléments de lexicographie gabonaise, Tome I: 7-37. New York: Jimacs-Hillman.

Mission Catholique du Gabon. 1925. Syllabaire Fang. Mellville (Nord): Imprimerie Ceugnard Le sage.

Mission Évangélique de Paris. 1927. Catéchisme Élémentaire en Fang. Réédité en 1931 et 1958 par la Société des Missions Évangéliques de Paris.

Mission Protestante du Congo Français. 1912. Ntèn Fan Ôsua: Premier Livre de Lecture en Fan. SaintBlaise (Suisse): Samuel Robert.

Mission Protestante de Talagouga. 1889. Nteni osu Nteni Fanwe. Talagouga/Ndjolé.

Mission Protestante de Talagouga. 1898. Nten Misili Ye biya bi fan Ôsua. Talagouga.

Mission Protestante Française. 1920. Ezangô Elônga Zi Galwa: Premier Livre de Lecture en Galoa. London: A.E.F. 
Muroni, J.-M. 1989. Petit dictionnaire du bantu du Gabon. Paris: l'Harmattan.

Nsuka Nkutsi, F. (Éd.). 1980. Éléments de Description du Punu. Lyon: Université de Lyon II.

Ondo Mebiame, P. 1992. De la phonologie à la morphologie du Fang parlé à Aboumezok (langue Bantu A.78). 2 volumes. Thèse de doctorat. Tervuren: Université Libre de Bruxelles.

Puech, G. 1990. Bekwil. Revue Gabonaise des Sciences de l'Homme 2: 127-128.

Raponda Walker, A. et R. Sillans. 1961. Plantes utiles: essai d'inventaire et de concordance des noms vernaculaires et scientifiques des plantes spontanées et introduites du Gabon. Paris: Lechevalier.

Raponda Walker, A. 1995². Dictionnaire Français-Mpongwé. Class. Africaines.

Raponda Walker, A. 1995². Dictionnaire Mpongwé-Français. Class. Africaines.

Raponda Walker, A. 1995. Éléments de grammaire Fang. Libreville: Éd. Raponda.

Raponda Walker, A. 1995. Éléments de grammaire Gisira. Libreville: Éd. Raponda.

Raponda Walker, A. 1995. Essai de grammaire Tsogo. Livreville: Éd. Raponda.

Voltz, M. 1990. Fan (Ntumu). Revue Gabonaise des Sciences de l'Homme 2.

Warmelo, N.J. van. 1937. Tshivenda-English Dictionary. Pretoria: Government Printer. 